\title{
Subject Index to Volume 23
}

Acromegaly, somatomedin values in urine, 151

Acyl-coenzyme A dehydrogenase, longchain, deficiency, fibroblast studies, 603

$\beta$-Adrenoreceptor, stimulation and contractile state, role, patent ductus arteriosus, preterm lamb, 316

Age, indomethacin effects and, hypoxic vasoconstriction, neonatal lamb lung, 580

Airway

activity, surface cooling effect, lamb, 257 immature, ventilation effect, 519

pressure, increasing, high-frequency oscillatory ventilation and, 628

Alanine, blood concentrations, effect of fasting, rat, 241

Aluminum, hepatobiliary dysfunction related to, rat, 275

Alveolar fluid, changes at birth, term and premature lamb, 418

Alveoli, macrophage, status, bronchopulmonary dysplasia, 470

Amino acids, calcium-sensitive uptake, by human placental slices, 9

Anemia, chronic, cardiovascular adaptations, newborn lamb, 621

Antibiotics, effect on intestinal heme oxygenase, rat, 50

Antibody, longitudinal development, very low birth weight premature infants, 14

Apnea, graded duration, cardiovascular and neurophysiologic changes, piglet, 402

Apolipoprotein A-1, in umbilical cord blood, gestational age and, 348

Arousal response, repeated upper airway obstruction and, neonatal lamb, 19

Atenolol, effects on growth hormone release, constitutional delay of growth, prepubertal boys, 393

Atopy, infantile recurrent otitis media and, 509

Audiometry, reflex modification, acoustic sensory processing assessed by, neonate, 357

Bacteremia, Gram-positive, thromboxaneassociated pulmonary hypertension during, piglet, 553

Bile acid

efflux, from suckling rat hepatocytes, 364

uptake from terminal ileum, cystic fibrosis, 323

Bilirubin

conjugation and production, effect of tinprotoporphyrin, cholestatic rat, 163

effects on brain energy metabolism, during normoxia and hypoxia, 569

short-term exposure, synaptic activation reduced by, rat brain, 453

synapsin I phosphorylation, rat cerebral cortex, 219

uptake, role of free bilirubin acid salt, 443

Birth, pulmonary alveolar subphase changes, term and premature lamb, 418

Blood flow

cerebral

alterations, during and after partial ischemia, piglet, 206 velocity, spectral analysis, premature infants, 398

Blood pressure

apnea effect, piglet, 402

caffeine effect, rat and chick embryo, 200

ethnic variation, preadolescent children, 270

spectral analysis, premature infants, 398

Brain

cerebral metabolism, newborn lamb with polycythemia, 329

energy, metabolism, bilirubin effects, during normoxia and hypoxia, 569

glucose metabolism, euglycemic hyperinsulinemia and, newborn dog, 474

synaptic activation in transverse hippocampal slices, bilirubin exposure and, rat, 453

Brainstem, auditory evoked potential, abnormalities, jaundiced Gunn rat, 306

Bronchopulmonary dysplasia

alveolar macrophage status in, 470

water balance control, role of endogenous vasopressin, infants, 86

Caffeine

hemodynamic changes and, rat and chick embryo, 200

$\mathrm{N}$-demethylation, maturation in infancy, 632

Calcium

absorption, measurement, premature infants, 589

role in cellular regulation of amino acid transport, 9

Capillary, blood cell velocity, videophotometric microscopic determination, infants, 585

Cardiac-sympathetic axis, role in cardiac growth, neonatal nutritional deprivation or enhancement, rat, 423

Carnitine, deficiency, fasting free fatty acids and, 491

Catecholamin

conjugated versus free acidic metabolites, neuroblastoma diagnosis, 576

contractile state and, patent ductus arteriosus, preterm lamb, 316

Catheterization, glucose and lactate changes, acute versus chronic catheterized rats, 235

Chloramphenicol, pharmacokinetics, effect of exocrine pancreatic function, cystic fibrosis, 388

Cholecystokinin-octapeptide, effect on amylase activity, fetal pancreas, 539

Cholestasis, bilirubin conjugation and production, effect of tin-protoporphyrin, rat, 163

Cholesterol, high-density lipoprotein, apolipoprotein A-1 and, 348

Chorionic gonadotropin, human, luteinizing hormone and, dynamics, first 7 days of life, 530

Circulation, arterial and portal venous, galactose, glucose, and lactate concentrations after nursing, newborn lamb, 598

Coagulation, neonatal, fibrinogen turnover, lamb, 249

Collagen gel, motility of human milk macrophages in, 449

Cromolyn sodium, aerosolized, pulmonary vascular response, lamb, 513

Cysteamine, effectiveness in nephropathic cystinosis, compared with phosphocysteamine, 616

Cystic fibrosis

bile acid uptake from terminal ileum in 323

chloramphenicol pharmacokinetics, effect of exocrine pancreatic function, 388

Cystinosis, nephropathic, comparison, cysteamine and phosphocysteamine effectiveness, 616

Dazmegrel, streptococcal group B sepsis, effects of late treatment, neonate, 352

Dehydration, cellular, senescent neonatal erythrocytes, 288

Diabetes, offspring of affected parents, thiamin status, 574

Diaphragm

electromyographic activity

effect of hypercapnia and hypoxia, piglet, 54

obstructive sleep apnea, 1

unilateral paralysis, postnatal lung growth inhibited by, piglet, 463

Diarrhea, viral, glucocorticoid effect on jejunum during, piglet, 279

Dichloroacetate, skin fibroblast culture, pyruvate metabolism disorders detected by, 561

Digitalis

receptor

erythrocytes, preterm infants and adults, 414

Dipalmitoyl phosphatidylcholine, pulmonary surfactant and, in vitro and in vivo effects, 484

DNA, synthesis, aspirin-like drug effects,

Drugs pregnant rats and offspring, 93

antibiotic, effect on intestinal heme oxygenase, rat, 50

aspirin-like, effect on lymphocyte transformation, pregnant rats and offspring, 93

Electroencephalogram, apnea effect, piglet, 402

Embryo, developmental hemodynamic changes, caffeine effect, chick and rat, 200

Enteral feeding, see Nutrition

Enteritis

bacterial, malnutrition effects on intestinal repair after, infant rabbit, 408

viral, glucocorticoid effect on jejunum during, piglet, 279

Enzyme

intestinal, development, infant miniature pig, 311

pancreatic, reserpine effect on adaptive response to diet, rat, 176

Epidermal growth factor 
effect on amylase activity, fetal pancreas, 539

receptor ontogeny, hepatic, neonatal hyperthyroidism and, mouse, 557

Epinephrine

release, naloxone effect, during hypoxia, fetal sheep, 343

renal vascular effect, fetal, newborn, and adult sheep, 181

Erythrocyte

bilirubin uptake, role of free bilirubin acid salt, 443

digitalis receptor in, comparison, preterm infants and adults, 414

senescent neonatal, cellular dehydration and immunoglobulin binding in, 288

Erythropoiesis, biosynthetic insulin-like growth factor-1 effect, neonatal rat, 298

European Society for Paediatric Endocrinology, abstracts of annual meeting, 105

Fasting, effect on glucose, lactate, alanine, and glutamate concentrations, rat, 241

Fatty acid

catabolism, effect of salicylic acid, 338

composition, intestinal microvillus membrane, newborn and adult rat, 439

free, fasting, effect of carnitine deficiency, 491

oxidation

control, small intestine, suckling rat, 262

Fetus liver, postterm rabbit fetus, 224

airway activity, surface cooling effect, lamb, 257

breathing movements, effect of thyrotropin-releasing hormone, sheep, 72

conversion of thyroxine to $3,3^{\prime}, 5^{\prime}$-triiodothyronine, rat, 196

development, apolipoprotein A-1 during, 348

epinephrine effect, renal vascular bed, sheep, 181

glucose utilization

effects of insulin and glucose concentrations, lamb, 381

fed and fasted pregnant rabbits, 480

heart rate during hypoxia, transcutaneous monitoring, dog, 548

hypoxemia, renal hemodynamic responses to, lamb, 155

hypoxia, naloxone effect on epinephrine release, lamb, 343

lung compliance, pulmonary surfactant effect, rabbit, 23

pancreas, amylase activity, effect of cholecystokinin-octapeptide, insulin, glucagon, triiodothyronine, and epidermal growth factor, 539

protein turnover, after maternal malnutrition, rat, 534

substrate utilization, last trimester, sheep lung, 606

Fibrinogen, turnover, newborn lamb, 249

Fibroblast

human skin, metabolism of branched chain 2-oxo acids, 40

long-chain acyl-coenzyme A deficiency, biochemical studies, 603

skin, culture, pyruvate metabolism disorders detected by, 561

Galactose, in portal venous and arterial circulations, after nursing, newborn lamb, 598
Gastrointestinal tract, intestinal absorption of lead, suckling rat, 58

Genioglossus, electromyographic activity, obstructive sleep apnea, 1

Glucagon, effect on amylase activity, fetal pancreas, 539

Glucocorticoid

effect on jejunum, during acute viral enteritis, piglet, 279

plasma, premature infants, first week of life, 525

Gluconeogenesis, premature appearance, liver, postterm rabbit fetus, 224

Glucose

blood concentrations

effect of fasting, rat, 241

effect on utilization, fetal lamb, 381

in portal venous and arterial circulations, after nursing, newborn lamb, 598

metabolism, euglycemic hyperinsulinemia and, cerebral cortex, neonatal dog, 474

portal venous and aortic changes, chronically catheterized rat, 235

utilization

effects of insulin and glucose concentrations, fetal lamb, 381

placenta and fetal tissues, fed and fasted pregnant rabbits, 480

Glutamine, blood concentrations, effect of fasting, rat, 241

Glycogen storage disease, type $1 \mathrm{~A}$, liver metabolism in, 375

Growth and development

constitutional delay, atenolol effects on growth hormone release, prepubertal boys, 393

diastolic ventricular interaction and, 466

effect of protein-calorie malnutrition on developing liver, 505

excitation-contraction coupling, developing myocardium, rabbit, 428

immunoreactive somatomedin $\mathrm{C}$ detection in urine, normal subjects, pituitary dwarfs, acromegalics, 151

phosphate transport by intestinal endoplasmic reticulum, during maturation, 612

urinary growth hormone measurement, children with normal and abnormal growth, 89

ventilation effect on immature airways, 519

Growth hormone

atenolol effects, constitutional delay of growth, prepubertal boys, 393

recombinant human, treatment monitoring, 167

urinary excretion, quantitation, normal and abnormal growth, 89

Heart

atrioventricular nodal function, immature canine heart, 99

diastolic ventricular interaction, developmental changes, 466

myocardial dysfunction, $\mathrm{HCl}$-induced, endogenous opioids and, 643

myocardial excitation-contraction coupling, developing rabbit, 428

pacemaker development, embryonic rat, 637

ventricle, diastolic interaction, developmental changes, 466

Heart rate

apnea effect, piglet, 402

during hypoxia, transcutaneous monitoring, dog, 548
Heme oxygenase, intestinal, effects of antibiotics and tin-protoporphyrin, 50

Hemoglobin, switching, rate estimation by measuring hemoglobin F,neonatal reticulocyte, 595

Hepatocyte, bile acid efflux from, suckling rat, 364

Hepatoma cells, human, influenza infected, protein synthesis by, 334

Heterozygote, detection, Pompe's disease, 283

Hypercapnia, effect on diaphragm electromyogram, piglet, 54

Hypercholesterolemia, familial, screening, neonates, 500

Hyperinsulinemia, euglycemic, effect on cerebral cortical glucose metabolism, neonatal dog, 474

Hypernatremia, chronic, role of taurine as cerebral osmoprotective molecule, kitten, 35

Hyperphenylalaninemia, mouse mutant, 63

Hypertension, pulmonary, thromboxane associated, Gram-positive bacteremia, piglet, 553

Hyperthyroidism, neonatal, hepatic epidermal growth factor receptor ontogeny and, mouse, 557

Hyperventilation, whole body oxygen consumption and, newborn piglet, 565

Hypoxanthine, as hypoxia indicator, role in health and disease, 143

Hypoxemia

acute, effect of $\beta$-adrenergic receptor blockade, lamb, 229

chronic, comparison, chronic anemia, newborn lamb, 621

renal hemodynamic responses, relationships to circulating vasoactive substances, fetal and neonatal lamb, 155

Hypoxia

brain energy metabolism during, bilirubin effects, 569

effect on diaphragm electromyogram, piglet, 54

epinephrine release during, naloxone effect, fetal sheep, 343

fetal heart rate during, transcutaneous monitoring, dog, 548

hypoxanthine as indicator of, 143

isocapneic alveolar, pulmonary vascular response, lamb, 513

Ileum, terminal, bile acid uptake from, cystic fibrosis, 323

Immunoglobulin, binding, senescent neonatal erythrocytes, 288

Indomethacin, age-dependent effects on hypoxic vasoconstriction, neonatal lamb lung, 580

Infant

see also Neonate

bacterial enteritis, malnutrition effects on intestinal repair after, rabbit, 408

bronchopulmonary dysplasia, control of water balance, 86

capillary blood cell velocity, videophotometric microscopic determination, infants, 585

group B Streptococcus, type III, antibody activity, 31

intestinal enzyme development, miniature pig, 311

low birth weight, vitamin $\mathrm{E}$ effect on superoxide production, 245

lung lavage cell phenotype, sudden infant death syndrome, 187 
maturation of caffeine $\mathrm{N}$-demethylation, 632

premature

digitalis receptor in erythrocytes from, 414

high-density lipoprotein subclass distribution, before and after enteral feeding, 543

spectral analysis of cardiovascular instability, 398

recurrent otitis media, risk factors, 509

very low birth weight

antibody development, 14

lymphocyte proliferative response subpopulations in, 457

Influenza B, human hepatoma cells infected with, protein synthesis, 334

Insulin

concentration, effect on glucose utilization, fetal lamb, 381

effect on amylase activity, fetal pancreas, 539

Insulin-like growth factor-1, biosynthetic, effects on somatic growth, maturation, and erythropoiesis, neonatal rat, 298

Intestine

endoplasmic reticulum, phosphate transport by, during maturation, 612

microvillus membrane phospholipids in, newborn and adult rat, 439

Iron, nonheme, isotope determination, 495

Ischemia, partial, cerebral blood flow alterations, piglet, 206

Jaundice, acute brainstem auditory evoked potential abnormalities in, Gunn rat given sulfonamide, 306

Jejunum, mucosa, glucocorticoid effect, during viral enteritis, piglet, 279

\section{Kidney}

hemodynamic response to hypoxemia, fetal and neonatal lamb, 155

vascular bed, epinephrine effects, fetal, newborn, and adult sheep, 181

Lactate

blood concentrations, effect of fasting, rat, 241

in portal venous and arterial circulations, after nursing, newborn lamb, 598

portal venous and aortic changes, chronically catheterized rat, 235

Latin American Society for Pediatric Research, abstracts, 647

Lead, distribution in milk and intestinal contents, suckling rat, 58

Lipoprotein, high-density, subclass distribution, before and after enteral feeding, premature neonate, 543

Liver

dysfunction, aluminum-associated, rat, 275

epidermal growth factor receptor ontogeny, neonatal hyperthyroidism and, mouse, 557

gluconeogenesis, premature appearance, postterm rabbit fetus, 224

immature, effect of protein-calorie malnutrition, 505

metabolism, glucose-6-phosphatase deficiency, 375

Lung

aurine transport, suckling rat, 172

alveolar subphase, changes at birth, term and premature lambs, 418 fetal, substrate utilization, last trimester, lamb, 606

hypoxic vasoconstriction, age-dependent indomethacin effects, 580

lavage, cell phenotype, sudden infant death syndrome, 187

postnatal growth, unilateral diaphragmatic paralysis and, piglet, 463

pulmonary oxygen toxicity, vitamin A deficiency and, mouse, 76

response to aerosolized cromolyn sodium and isocapneic alveolar hypoxia, lamb, 513

Luteinizing hormone, human chorionic gonadotropin and, dynamics, first 7 days of life, 530

Lymphocyte

proliferative response subpopulations, analysis, very low birth weight infants, 457

regulation by human placental cells, 212

transformation, effect of aspirin-like drug ingestion during pregnancy, rat, 93

zinc-stimulated, heterozygote detection, Pompe's disease, 283

\section{Macrophage}

alveolar

function, neonatal and juvenile rhesus monkey, 293

status, bronchopulmonary dysplasia, 470

human milk, motility, collagen gels, 449

Magnetic resonance spectroscopy, liver metabolism studied by, glucose-6-phosphatase deficiency, 375

Malnutrition

maternal, protein turnover in fetal tissues after, rat, 534

protein-calorie

effect on developing liver, 505

small intestinal repair affected by, infant rabbit, 408

Metabolism

cerebral, newborn lamb with polycythemia, 329

gestational changes, pregnant guinea pig uterus, 45

Microscopy, videophotometric, capillary blood cell velocity determined by, infants, 585

Milk

human, macrophage motility, in collagen gels, 449

lead content, suckling rat, 58

Mineralocorticoids, plasma, premature infants, first week of life, 525

Mitochondria, bilirubin uptake, role of free bilirubin acid salt, 443

Myocardium

dysfunction, $\mathrm{HCl}$-induced, endogenous opioids and, 643

excitation-contraction coupling, developing rabbit, 428

Naloxone, epinephrine release potentiated by, during hypoxia, fetal sheep, 343

Neonate

see also Infant

acoustic sensory processing, reflex modification audiomety assessment, 357

acute hypoxemia, effect of $\beta$-adrenergic receptor blockade, lamb, 229

altered nutritional status, role of cardiacsympathetic axis, rat, 423

atrioventricular nodal function, puppy, 99 biosynthetic insulin-like growth factor- 1 effect, rat, 298

cerebral blood flow alterations, during and after partial ischemia, piglet, 206

chronic anemia, cardiovascular adaptations, lamb, 621

coagulation, fibrinogen turnover, lamb, 249

dynamics of bioactive luteinizing hormone-human chorionic gonadotropin, 530

epinephrine effect, renal vascular bed, lamb, 181

euglycemic hyperinsulinemia, glucose metabolism and, cerebral cortex, dog, 474

familial hypercholesterolemia screening, recall study, 500

group B streptococcal sepsis, late dazmegrel treatment, 352

hepatic taurine transport, rat, 172

hyperthyroidism, hepatic epidermal growth factor receptor ontogeny and, mouse, 557

hypoxemia, renal hemodynamic responses to, lamb, 155

hypoxic vasoconstriction, age-dependent indomethacin effects, lamb lung, 580

intestinal microvillus membrane, fatty acid composition, rat, 439

lymphocyte transformation, effect of aspirin-like drug ingestion during pregnancy, rat, 93

ontogeny of pulmonary defenses, rhesus monkey, 293

oxygen consumption, hyperventilation and, piglet, 565

polycythemia, cerebral metabolism in, lamb, 329

portal venous and arterial circulations, galactose, glucose, and lactate concentrations, after nursing, lamb, 598

premature, high-density lipoprotein subclass distribution, before and after enteral feeding, 543

repeated upper airway obstruction, effect on arousal and cardiopulmonary response, lamb, 191

reticulocyte, hemoglobin switching rate estimation, 595

Neuroblastoma, diagnosis, catecholamine metabolites, random urine samples, 576

Neurotoxicity, bilirubin and synapsin I phosphorylation, rat cerebral cortex, 219

Nicotinamide adenine dinucleotide, fatty acid oxidation controlled by, small intestine, suckling rat, 262

Nitrogen, washout, changes in ventilation homogeneity and, 68

Normoxia, brain energy metabolism during, bilirubin effects, 569

Nutrition

effect of protein-calorie malnutrition on developing liver, 505

enteral, high-density lipoprotein subclass distribution and, premature neonate, 543

infantile recurrent otitis media and, 509

neonatal, role of cardiac-sympathetic axis, rat, 423

nonheme iron, stable isotope determination, 495

parenteral, aluminum-associated hepatobiliary dysfunction, 275

Opioid, endogenous, effect on $\mathrm{HCl}$-induced myocardial dysfunction, 643 
Otitis media, infantile recurrent, risk factors, 509

2-Oxo acids, branched chain, metabolism, human skin fibroblasts, 40

Oxygen

consumption, hyperventilation and, newborn piglet, 565

pulmonary, toxicity, vitamin A deficiency and, mouse lung, 76

saturation, apnea effect, piglet, 402

tension, inner retinal surface, rabbit, 5

Pacemaker, development, embryonic rat heart, 637

Pancreas

exocrine

function, effect on choramphenicol pharmacokinetics, cystic fibrosis, 388 reserpine effect on dietary adaptation, rat, 176

fetal, amylase activity, effect of cholecystokinin-octapeptide, insulin, glucagon, triiodothyronine, and epidermal growth factor, 539

Paralysis, diaphragmatic, postnatal lung growth and, piglet, 463

Parents, diabetic, thiamin status of offspring, 574

Patent ductus arteriosus, role of $\beta$-adrenoreceptor stimulation and contractile state, preterm lamb, 316

Phosphate, transport, by intestinal endoplasmic reticulum, during maturation, 612

Phosphocysteamine, effectiveness in nephropathic cystinosis, compared with cysteamine, 616

Phospholipid, microvillus membrane, small intestine, newborn and adult rat, 439

Pituitary dwarfism, somatomedin values in urine, 151

Placenta

calcium-sensitive amino acid uptake, 9

glucose utilization, fed and fasted pregnant rabbits, 480

immunologic function, 212

Plethysmography, impedance, tidal volume measured by, 253

Polycythemia, neonatal, cerebral metabolism, lamb, 329

Polymorphonuclear leukocyte, superoxide anion production, vitamin $\mathrm{E}$ effect, 245

Pompe's disease, heterozygote detection, enzyme assay method, 283

Postmaturity, liver metabolism and, rabbit fetus, 224

Pregnancy

aspirin-like drug ingestion during, effect on lymphocyte transformation, rat, 93

glucose utilization, fed and fasted pregnant rabbits, 480

uterine metabolism, function of gestational age, guinea pig, 45

Prematurity

see also Infant, premature

calcium absorption, measured by intravenous ${ }^{46} \mathrm{Ca}$ and oral ${ }^{44} \mathrm{ca}, 589$

patent ductus arteriosus, role of $\beta$-adrenoreceptor stimulation and contractile state, lamb, 316

plasma mineralocorticoids, glucocorticoids, and progestins, first week of life, 525

pulmonary surfactant effect on lung compliance, rabbit fetus, 23

specific and functional antibody, longitudinal development, 14
Procollagen type III, in human growth hormone treatment, long-term monitoring, 167

Progestin, plasma, premature infants, first week of life, 525

Propranolol, effects during hypoxemia, newborn lamb, 229

Protein

synthesis, human hepatoma cells infected with influenza B virus, 334

turnover, fetal tissue, after maternal malnutrition, rat, 534

Pulmonary surfactant

dipalmitoyl phosphatidylcholine and, in vitro and in vivo effects, 484

effect on lung compliance, premature rabbit fetus, 23

Pyruvate, metabolism, disorders detected with dichloroacetate culture of skin fibroblasts, 561

Race, blood pressure and, ethnic variation preadolescent children, 270

Reserpine, effect on dietary adaptation, rat exocrine pancreas, 176

Respiration, thyrotropin-releasing hormone effect, fetal sheep, 72

Reticulocyte, neonatal, hemoglobin switching rate estimation, 595

Reticulum, intestinal endoplasmic, phosphate transport by, during maturation, 612

Retina, physiological effects of light and oxygen, retinopathy of prematurity, rabbit, 5

Reye's syndrome

pathogenesis

role of influenza $\mathrm{B}, 334$

role of salicylic acid, 338

Salicylic acid, effect on fatty acid catabolism, 338

Screening, familial hypercholesterolemia, neonates, 500

Sepsis, streptococcal group B, late dazmegrel treatment, neonate, 352

Serotonin, turnover, effects of sodium benzoate and sodium phenylacetate, child with citrullinemia and animal model, 368

Skin

capillary blood cell velocity, videophotometric microscopic determination, infants, 585

fibroblast, dichloroacetate detection of pyruvate metabolism disorders by culture of, 561

human fibroblasts, metabolism of branched chain 2-oxo acids, 40

Sleep apnea, obstructive, diaphragmatic and genioglossus electromyographic activity, 1

Small intestine

fatty acid oxidation, control by [NADH/ $\mathrm{NAD}^{+} /$], suckling rat, 262

repair, after bacterial enteritis, malnutrition effect, infant rabbit, 408

Sodium benzoate, effect on brain serotonin turnover, child with citrullinemia and animal model, 368

Sodium phenylacetate, effect on brain serotonin turnover, child with citrullinemia and animal model, 368

Somatomedin, biosynthetic, effects on somatic growth, maturation, and erythropoiesis, neonatal rat, 298

Somatomedin C, immunoreactive, urine, normal subjects, pituitary dwarfs, acromegalics, 151

Spectral analysis, blood pressure and cerebral blood flow velocity measured by, premature infants, 398

Spectroscopy, nuclear magnetic, effects of bilirubin on brain energy metabolism, 569

Streptococcus

group B

hemodynamic response, conscious piglet, 81

sepsis, late dazmegrel treatment, neonate, 352

group B, type III, class-specific antibody activity, infants, 31

Substrate, utilization, last trimester, fetal sheep lung, 606

Sudden infant death syndrome, lung lavage cell phenotype in, 187

Superoxide, production, decreased by vita$\min \mathrm{E}, 245$

Synapsin I, phosphorylation, bilirubin effect, rat cerebral cortex, 219

Taurine

hepatic transport, suckling rat, 172

role as cerebral osmoprotective molecule, chronic hypernatremia, kitten, 35

Taurocholate, efflux, from suckling rat hepatocyts, 364

Thermoreceptor, cutaneous, airway activity and surface cooling, fetal lamb, 257

Thiamin, in offspring of diabetic rats, 574

Thromboxane, pulmonary hypertension associated with, during Gram-positive bacteremia, piglet, 553

Thyroglobulin, secretion control, ectopic thyroid gland patients, 266

Thyrotropin-releasing hormone, respiratory and electocortical effects, fetal sheep, 72

Thyroxine, conversion to $3,3^{\prime}, 5^{\prime}$-triiodothyronine, fetal rat tissue, 196

Tidal volume, measurement, during high frequency ventilation, 253

Tin-protoporphyrin

effect on bilirubin conjugation and production, cholestatic rat, 163

effect on intestinal heme oxygenase, rat, 50

Triiodothyronine, effect on amylase activity, fetal pancreas, 539

Tryptophan, turnover, effect of sodium benzoate, and sodium phenylacetate, child with citrullinemia and animal model, 368

Upper airway, repeated obstruction, arousa and cardiopulmonary response, lamb 191

Urine

growth hormone excretion, children with normal and abnormal growth, 89

immunoreactive somatomedin $\mathrm{C}$ in, normal subjects, pituitary dwarfs, acromegalics, 151

neuroblastoma diagnosis, catecholamine metabolites and, 576

Uterus, metabolism, function of gestational age, pregnant guinea pig, 45

Vasoconstriction, hypoxic, age-dependent effects of indomethacin, neonatal lamb lung, 580

Vasopressin, endogenous, role in broncho- 


\section{SUBJECT INDEX TO VOLUME 23}

pulmonary dysplasia, infants, 86 Ventilation

effect on immature airways, 519

high frequency, tidal volume measurement during, 253

high-frequency oscillatory, hemodynamics impaired during, 628

homogeneity changes, growth effect, 68 Ventriculotomy, right, effects on local propagation, puppy, 433

Vitamin A, deficiency, pulmonary oxygen toxicity and, mouse lung, 76
Vitamin E, superoxide production decreased by, 245

Water balance, control, vasopressin role, infants with bronchopulmonary dysplasia, 86 